\title{
Renal Pathophysiology
}

\author{
Siniša Franjić \\ Indenpendent Researcher, Croatia \\ *Corresponding Author: Siniša Franjić, Independent Researcher, Croatia.
}

\begin{abstract}
Pathological conditions of the kidney may be due to disorders that primarily affect blood vessels, glomeruli, tubules, and interstitial tissue that separates individual nephrons. Various factors that can be divided into prerenal and postrenal can cause kidney disease or impaired renal function. Numerous prerenal factors can reduce renal blood perfusion to such an extent that they lead to secondary renal dysfunction. In a similar way, kidney disease can occur due to the action of postrenal factors that lead to obstruction of the urinary tract.
\end{abstract}

Keywords: Kidney, Blood, Blood Pressure, Pathophysiology

\section{INTRODUCTION}

The pathophysiology of acute kidney injury involves a complex interplay among vascular, tubular, and inflammatory factors followed by a repair process that can either restore epithelial differentiation and function to normal or result in progressive fibrotic chronic kidney disease [1]. Innate and acquired immunity play an important role in the injury phase, in the regulation of the inflammatory response, and in processes related to repair of the epithelial layer. Recent data change the direction of focus of the role of the epithelium in fibrosis and attributes myelofibroblast production to perivascular and interstitial fibroblasts. The epithelium plays an important role in abnormal repair through a recently defined link between cell cycle arrest of the epithelial cell and profibrogenic cytokine production.

Chronic kidney disease has become a global public health problem with an increasing prevalence and high mortality [2]. Owing to the growing elderly population and the increasing prevalence of hypertension and diabetes as well as the improving treatment strategies, the prevalence of CKD will inevitably continue to increase in the near future. Glomerular filtration rate (GFR) and albuminuria are proposed as the best indicators of kidney function, with low GFR and increased albuminuria being associated with a high risk of kidney failure requiring renal replacement therapy and of cardiovascular disease, anemia, mineral and bone disorder, and other complications.

Many patients with CKD follow a predictable clinical course following disease initiation, with progressive renal dysfunction ultimately resulting in ESKD (end-stage kidney disease) [3]. Critically, CKD is clinically silent in up to 90\% patients until it has reached an advanced stage, and patients who reach ESKD without prior contact with nephrology services experience greater co-morbidity and poorer survival following initiation of renal replacement therapy. There is therefore an opportunity to detect patients with asymptomatic CKD by screening, with the aim of applying therapies to ameliorate disease progression.

\section{BLOOD}

Such a result may occur when there is a genuine increase in red blood cells in the urine or may be a consequence of the presence of an abnormal amount of the pigment haemoglobin in the urine [4]. Haemoglobin is the pigment which gives blood its red colour and which is responsible for the carriage of oxygen within red blood cells. Haemoglobinuria occurs when there is rapid destruction of red cells within the circulation (haemolysis) in, for example, malaria, after trauma or snake bite. A positive result on stix testing should prompt examination of a fresh urine sample under the microscope. The absence of an excess of red blood cells suggests the presence of haemoglobinuria. Error may be introduced if the urine is examined many hours after it has been passed, as red cells break down with time and may no longer be discernible.

Blood in urine does not invariably indicate that it has issued from the urinary tract. A major trap for the unwary is to find a positive stix test result in a woman who is menstruating. The 
institution of detailed investigations, some of which carry risk, under such circumstances would be negligent. The finding of blood in urine which is not discernible to the naked eye is termed 'microscopic haematuria'. Further investigation is mandatory. The causes are many and include bladder and kidney tumours, urinary tract stones and immunologically mediated disease of the filtering units of the kidney (glomeruli), the general term for which is 'glomerulonephritis'. Investigation will include in many cases a search for malignant cells in urine under the microscope, assessment of renal function, imaging of the urinary tract and perhaps direct inspection of the bladder via a cystoscope introduced through the urethra, the passage leading from the bladder to the exterior. Cystoscopy is usually the province of the urologist rather than the nephrologist.

Blood pressure is a direct function of cardiac output and peripheral vascular resistance [5]. Cardiac output is directly related to the heart rate and stroke volume. Because his heart rate was rapid, one should look for a process that could compromise his stroke volume and/or possibly lower his peripheral resistance. Because he did not have evidence of blood loss, sepsis, or a disorder that can cause salt deficiency (e.g., a history of vomiting, diarrhea), suspect that there is a problem with contractility of his heart and/or the vasoconstrictor tone of his blood vessels. One factor that is essential for contractility is ionized calcium. Therefore it is possible that the new anion might have removed ionized calcium. Because citrate chelates ionized calcium (e.g., this is why citrate is used to prevent coagulation of the blood), this patient may have ingested citric acid. As part of the emergency treatment of hyperkalemia, he was given a bolus of a calcium salt. This corrected the low concentration of ionized calcium in plasma, and hence his myocardial contractility increased, as did his peripheral vascular resistance. This was another piece of the puzzle that alerted his physicians to the possibility that this patient may have ingested citric acid. This was confirmed later when the composition of the ingested solution became known.

\section{CKD}

Patients with chronic kidney disease(CKD) are two to four times as likely to have cardiovascular disease (CVD) compared to the general population, when adjusted for traditional CVD risk factors [6]. CVD is the leading cause of death in these patients, with vascular and valvular calcification being an integral part of its pathophysiology. Calcium phosphate crystals are deposited through a multifactorial dynamic process that leads to the development of atherosclerosis, arteriosclerosis, and valvular calcification.

Vascular calcification can be classified into intimal calcification and medial calcification according to the location of calcium deposition within the arterial wall. Intimal calcification is commonly associated with atherosclerotic plaques that partially occlude the arterial lumen, reducing blood flow and resulting in peripheral ischemia, myocardial infarction, stroke, and sudden death. Alternatively, medial calcification is deposited circumferentially along the elastic lamellae, which damages the elastic collagen resulting in an increase in wall stiffness and a decrease in vascular compliance. Clinically, medial calcification is more commonly seen in patients who are older, diabetic, and those with CKD.

Valvular calcification is an independent predictor of CVD, heart failure, and death, and is often responsible for leaflet and annular thickening with resulting valve dysfunction (i.e., stenosis). Calcification is more common in the aortic and mitral valves due to the higher pressures, turbulence, and mechanical stress seen on the left side of the heart compared to the right side. In the aortic valve, the increased calcium deposition often causes aortic stenosis (AS). Though symptoms of AS (angina, syncope, and dyspnea) are identical in patients regardless of baseline kidney function, the natural course of the disease is accelerated in patients with CKD and as a result these rapid progressors have severe, symptomatic AS at a younger age than the non-CKD population.

Chronic kidney disease, a general term for heterogeneous disorders, frequently occurs in association with a variety of factors including diabetes, nephritis, hypertension, and immune system disorder [7]. As the etiologically distinct cause progresses, a common renal pathological manifestation including glomerulosclerosis and/or interstitial fibrosis develops regardless of the cause. Over the past several years, rapid progress in deciphering the cellular and molecular mechanisms have led to better understanding of pathophysiology of CKD and would make it possible to develop clinically effective anti-CKD therapies.

The pathological manifestation of CKD is the loss of renal cells and deposition of the 
extracellular matrix (ECM). Regardless of the initial insults, the progressive renal disease is characterized by morphological changes that comprise renal inflammation, glomerulosclerosis, tubular atrophy, tubulointerstitial fibrosis, and capillary rarefaction. The pathogenesis of renal fibrosis including glomerulosclerosis and interstitial fibrosis is a progressive process that ultimately leads to end-stage renal failure, a devastating condition that requires renal replacement therapy (e.g., dialysis or transplantation). Current therapies for CKD mainly involve blockade of the renin-angiotensin-aldosterone system (RAAS). Thorough knowledge of the pathophysiology of kidney fibrosis is crucial for developing newer therapeutic strategies.

Chronic kidney disease has become a healthcare epidemic with increasing incidence and prevalence rates reported internationally [8]. It is estimated that approximately $3 \%$ of childbearing age women have CKD stages 1-2 and $0.7 \%$ have CKD stages 3-5. Although clear substantiating data is not available, it is the perception of the renal community that pregnancy rates in CKD, particularly in the later stages of $\mathrm{CKD}$, are less frequent when compared to the rates seen in women with normal renal function, and when pregnancies occur, they are considered to be high risk. The magnitude of this risk, however, is unclear. This is because most studies are small, do not report important outcomes such as maternal death, and include pregnancies in women with varying degrees of CKD caused by different underlying disorders with different comorbidities.

\section{CRS}

Cardiorenal syndrome (CRS) is a relatively new concept that acknowledges the complex interplay of heart failure and kidney failure [9]. As an entity, CRS carries with it a high mortality rate and significant medical resource utilization. In patients with heart failure and reduced kidney function of any magnitude, there is greater hospital resources use and readmissions . In patients with heart failure, creatinine clearances $(\mathrm{CrCl})<60 \mathrm{ml} / \mathrm{min}$ and changes in creatinine of greater than $0.3 \mathrm{mg} / \mathrm{dl}$ from baseline predict greater mortality when compared to patients with normal kidney function. Patients with chronic kidney disease (CKD), and/or a glomerular filtration rate (GFR) $<60 \mathrm{ml} / \mathrm{min} / 1.73 \mathrm{~m} 2$, have a higher risk of ischemic heart events, heart failure (preserved $\mathrm{EF}$ or reduced EF) and all cause death from cardiac causes than patients with normal GFR.
Theories that currently exist to explain the pathophysiologic interplay between the failing heart and kidneys involve the neurohumoral systems, which affect the body's sodium and volume homeostasis. Clinically the vicious cycle of heart failure and kidney failure that describes CRS is manifested by volume overload and resulting symptoms related to this: dyspnea, fatigue, and discomfort. Usual management of these symptoms is far more challenging in the CRS milieu because diuretic resistance, hypotension, hyperkalemia, and decreased renal perfusion are more prevalent than in heart failure with preserved renal function.

This scenario of high mortality, resource utilization, and high symptom burden suggests that patients with CRS would benefit from comanagement with Palliative Care. This approach to care focuses on symptom management and quality of life, and is often a part of the care of patients with heart failure or kidney failure. Symptom burden and psychosocial impact of chronic heart failure and chronic kidney disease are well defined in their individual disease territories, and less so in the overlap syndrome of CRS.

\section{Preeclampsia}

Preeclampsia, a leading cause of morbidity and mortality in pregnancy, complicates up to $5 \%$ of pregnancies worldwide [10]. The spectrum of hypertensive disorders during pregnancy comes in four varieties: gestational hypertension, preeclampsia and eclampsia syndrome, chronic hypertension, and preeclampsia superimposed on chronic hypertension. Gestational hypertension is the development of elevated blood pressure of greater than $140 / 90 \mathrm{mmHg}$ in previously normotensive women after 20 weeks of gestation. Proteinuria does not develop, and the elevated blood pressures resolve by 12 weeks postpartum. Preeclampsia is gestational hypertension with the presence of proteinuria and/or signs of end organ damage. The progression of the preeclampsia syndrome to eclampsia is signified by the onset of worsening hypertension and generalized tonic-clonic seizures. Preeclampsia and eclampsia are both definitively treated by delivery of the placenta, resulting in resolution of the acute syndrome. However, it has been shown that women who suffer from preeclampsia have a higher lifetime risk of cardiovascular disease (CVD), chronic kidney disease (CKD), cerebrovascular disease, and metabolic derangements including insulin 
resistance, overt diabetes mellitus, hyperlipidemia, and the metabolic syndrome.

While the clinical presentation is highly variable, risk of adverse outcomes increases significantly when preeclampsia develops early before 34 weeks of gestation [11]. Severe preeclampsia is characterized by severe hypertension (BP > 160/110 mmHg), evidence of end-organ damage, or intrauterine growth restriction. The most common presentation of preeclampsia is hypertension that is routinely detected at an antenatal visit in an asymptomatic woman. Most of the signs and symptoms will be present only in severe disease. The common symptoms in severe preeclampsia include vomiting, visual disturbances, headache, worsening of hand or leg edema, or severe persistent right upper quadrant or epigastric pain. On examination, in addition to higher blood pressures, patients can have papilledema, right upper quadrant tenderness, hyperreflexia with marked clonus, pulmonary edema, and altered mental status. Rapidly progressing signs and symptoms indicates impending severe disease that needs close monitoring. The major adverse outcomes include neurological complications such as seizures (eclampsia) and strokes, HELLP syndrome (defined by hemolysis, elevated liver enzymes, and low platelets), and renal dysfunction (ranging from mild reduction in glomerular filtration rate and minimal proteinuria to reversible or irreversible acute renal failure due to acute tubular necrosis or acute cortical necrosis). HELLP syndrome occurs in about $10-20 \%$ of women with severe preeclampsia and is associated with significant maternal and perinatal morbidity. Preeclampsia is a risk factor for cardiovascular disease, and preeclamptic women have 3.7 times higher risk of developing hypertension, 2.2 times increased risk of coronary heart disease, and 1.8 times higher risk of stroke. Although the absolute risk of end stage renal disease (ESRD) in women with history of preeclampsia is low, preeclampsia is associated with cumulative risk of subsequent ESRD.

\section{AlbuMinURIA}

The prevalence of kidney failure is increasing in the US and worldwide and is associated with poor outcomes and high costs [12]. During the last decade, much attention has been paid to early identification of individuals at risk for developing chronic kidney disease. The presence of albuminuria has emerged as an important risk marker of progressive renal functional loss in various populations. The importance of albuminuria as a diagnostic and prognostic risk marker has been recognized by policy and guideline makers, and albuminuria is currently included in a new CKD classification system in combination with estimated glomerular filtration rate (eGFR) to define the severity of CKD.

The glomerulus functions as a size-selective filter for protein filtration. Consequently, tubular fluid contains only proteins of low molecular weight $(<60 \mathrm{kD})$ such as vitamin D-binding protein or free retinol-binding protein, while larger proteins are excluded. Albumin, the most abundant plasma protein, is filtered in very low amounts $(1 \mathrm{e} 50 \mathrm{mg} / \mathrm{mL})$. In addition, albumin can be reabsorbed by the tubular epithelium. Consequently, normal total urinary protein excretion in the normal adult should be less than $150 \mathrm{mg} /$ day. 15 Higher rates of urinary protein excretion that persist beyond a single measurement should be evaluated. The increased protein leak can be caused by an increase in the circulating levels of several different proteins. For example, one may observe tubular proteinuria consisting of low molecular weight proteins (less than $25 \mathrm{kD}$ ). Such proteinuria usually reflects a systemic disease with overproduction of small peptide fragments that are filtered and may cause tubular damage (such as beta 2-microglobulin, immunoglobulin light chains, and polypeptides derived from the breakdown of albumin). In addition, interference with proximal tubular reabsorption, due to tubulointerstitial diseases or genetic mutations, or overproduction of immunoglobulins, such as occurs in myeloma, can lead to increased excretion of these smaller proteins.

\section{ESRD}

Patients with end-stage renal disease (ESRD) often suffer from cardiovascular complications and comorbidities [13]. For example, 55\% of ESRD patients suffer from congestive heart failure (CHF) and cardiovascular diseases account for the majority of deaths among ESRD patients. It is, therefore, of great importance to 
diagnose the underlying cardiac pathologies and to provide accurate risk stratification in ESRD patients.

Over the years, a number of accurate and sensitive biochemical markers have been introduced that have greatly advanced the diagnosis and risk stratification of cardiovascular diseases. The most prominent of these biochemical markers are the cardiac troponins (cTn, either $\mathrm{T}$ or $\mathrm{I}$ ) and the brain natriuretic peptides (BNPs) and their use has revolutionized the diagnosis and risk stratification of acute coronary syndromes (ACS) and CHF respectively. However, in the setting of ESRD, cardiac troponin concentrations can be elevated in the absence of apparent cardiac damage or clinical symptoms. Similarly, BNP and N-terminal proBNP (NTproBNP) concentrations are virtually always increased in ESRD patients. The presence of such continuously elevated cardiac troponin, BNP and NT-proBNP concentrations can interfere with their diagnostic and prognostic potential in ESRD patients.

\section{CONCLUSION}

Increasing the number of patients in all stages of kidney disease requires knowledge of the development and course of the disease, diagnosis and replacement therapy. Basic theoretical knowledge of the anatomy, physiology, and pathophysiology of the kidney is necessary as a basis for understanding disorders and the development of kidney disease. Knowledge of skills includes different treatment modalities such as: conservative treatment, peritoneal dialysis, hemodialysis, transplantation, and continuous replacement methods. Early patient involvement in educational programs and patient-centered care can reduce disease progression and the need for dialysis, reduce morbidity and mortality, correct anemia, prevent malnutrition with proper nutrition, promote self-care programs, and enable patient choice of method treatment.

\section{REFERENCES}

[1] Boventre, J. V. (2010.): „Pathophysiology of AKI: Injury and Normal and Abnormal Repair" in Ronco, C.; Bellomo, R.; McCullough, P. A.: „Cardiorenal Syndromes in Critical Care", Karger, Basel, Switzerland, pp. 9.
[2] Zhou, Y.; Yang, J. (2020.): „Chronic Kidney Disease: Overview" in Yang, J.; He, W. (eds): „Chronic Kidney Disease - Diagnosis and Treatment", Springer Nature Singapore Pte Ltd., Singapore, Singapore,pp. 3.

[3] Francis, R.; Johnson, D. (2012.): „Screening for Chronic Kidney Disease“ in Sahay, M. (ed): „Chronic Kidney Disease and Renal Transplantation“, InTech, Rijeka, Croatia,pp. 3.

[4] Baker, L. (1998.): „Nephrology“, Cavendish Publishing Limited, London, UK, pp. 20.

[5] Kamel, K. S.; Halperin, M. L. (2012.): „Metabolic Acidosis“ in Lerma, E. V.; Nissenson, A. R. (eds): „N Nephrology Secrets, Third Edition“, Mosby, Elsevier, Philadelphia, USA, pp. 589.

[6] Caughron, H.; Condado, J. F.; Babaliaros, V. (2017.): „Vascular and Valvular Calcification in Chronic Kidney Disease: Pathogenesis and Clinical Outcomes" in Rangaswami, J.; Lerma, E. V.; Ronco, C. (eds): , Cardio-Nephrology Confluence of the Heart and Kidney in Clinical Practice“, Springer International Publishing AG, Cham, Switzerland,pp. 11.

[7] Ren, J.; Dai, C. (2020.): „Pathophysiology of Chronic Kidney Disease" in Yang, J.; He, W. (eds): „Chronic Kidney Disease - Diagnosis and Treatment", Springer Nature Singapore Pte Ltd., Singapore, Singapore,pp. 13. - 14.

[8] Pahl, M. V. (2019.): „Pregnancy in Kidney Disease“ in Rhee, C. M.; Kalantar-Zadeh, K.; Brent, G. A. (eds): „Endocrine Disorders in Kidney Disease - Diagnosis and Treatment", Springer Nature Switzerland AG, Cham, Switzerland, pp. 139.

[9] Turner, R.; Shaban, H. (2017.): „Palliation with Dual Burden of Heart and Kidney Failure: An Underutilized Strategy" in Rangaswami, J.; Lerma, E. V.; Ronco, C. (eds): „CardioNephrology - Confluence of the Heart and Kidney in Clinical Practice", Springer International Publishing AG, Cham, Switzerland, pp. 205.

[10] Blumhof, S.; Rangaswami, J. (2017.): ”, Preeclampsia: A Mirror into Future Cardiovascular and Renal Health" in Rangaswami, J.; Lerma, E. V.; Ronco, C. (eds): „Cardio-Nephrology - Confluence of the Heart and Kidney in Clinical Practice", Springer International Publishing AG, Cham, Switzerland, pp. 385.

[11] Shah, S. (2020.): „Hypertensive Disorders in Pregnancy" in Sachdeva, M.; Miller, I. (eds): , Obstetric and Gynecologic Nephrology Women's Health Issues in the Patient With 
Kidney Disease“, Springer Nature Switzerland AG, Cham, Switzerland, pp. 15.

[12] Heerspink, H. J. L.; Rabelink, T.; de Zeeuw, D. (2020.): „Pathophysiology of Proteinuria: Albuminuria as a Target for Treatment"in Kimmel, P. L.; Rosenberg, M. E. (eds): , Chronic Renal Disease, Second Edition",
Academic Press, Elsevier, London, UK,pp. 211. -212 .

[13] Jacobs, L.; Mingels, A.; van Dieijen-Visser, M. (2012.): „ Cardiac Biomarkers in End-Stage Renal Disease“ in Sahay, M. (ed): „Chronic Kidney Disease and Renal Transplantation“, InTech, Rijeka, Croatia, pp. 147.

Citation: Siniša Franjić, “Renal Pathophysiology”, International Journal of Research Studies in Medical and Health Sciences. 2020; 5(10): 15-20.

Copyright: () 2020 Siniša Franjić, This is an open-access article distributed under the terms of the Creative Commons Attribution License, which permits unrestricted use, distribution, and reproduction in any medium, provided the original author and source are credited. 